\title{
Impulse Breakdown Characteristics of Main Gap in the Presence of a Local Discharge
}

\author{
A. Settaouti \\ Electrotechnic Department, University of Sciences and Technology, \\ P. O. Box. 1505 El-M'naouar, Oran - Algeria
}

\begin{abstract}
The characteristics of impulse breakdown voltages and the influence of the position of third electrode in air gap are investigated experimentally to study the parameters influencing the breakdown voltage in the presence of metallic objects around the high voltage power apparatus with air insulation. Experimental results show that the factors affecting the breakdown voltage are the shape and the size of the grounded electrode, the third metallic electrode location and the gap length. A comparison between negative and positive polarities of the applied voltages indicates an important influence of the polarity in the dielectric breakdown mechanism. The possible mechanism by which the local electric discharge initiates the main dielectric breakdown seems to be the high electric field around the local discharge channel and the streamers protruding from its surface.
\end{abstract}

Keywords: Breakdown, Electrical discharge; Electric field; High voltage; Triggered..

\section{Introduction}

In the high voltage and in the electrical equipments, the most of dielectrics used are the gases, research on the dielectric breakdown phenomena of the gaps insulated by gas are of a great interest. The electrical discharges in gases can be potentially implement in various applications like for example the deposition of films on substrate, the processes on the surfaces, in the bio-medical, the decontaminations and sterilization, the destruction of toxic constituents and sensors for the detection of trace of elements. The multiple gaps with electric discharges are employed in the systems of the high voltage electric power transmission, in the high voltage substations, for the high voltage equipments and for several applications. Due to the complexity of the process in the electrical discharges in the gases, there are multiples of question on the fundamental physical mechanism [1$10]$.

The lightning is an electrical discharge in the long gap can cause severe injuries to ground infrastructures. In the high voltage systems with multiples gaps arrangements, we have a high voltage electrode surrounded by several grounded devices; this causes the dielectric breakdowns on a long gap. Electrical discharges in gases represent a complex problem in the areas where the high-voltage techniques are utilised. For the improvements in the industries of the micro technology and nanotechnology, we must study the gap spacing between conductors. It is essential to understand the physical mechanism of the triggering process to ameliorate the geometry of the gaps. Therefore, it is necessitates studies of the mechanisms responsible for dielectric breakdown in gases.

\footnotetext{
* Corresponding author.

E-mail address: settaouti@hotmail.com
}

Manuscript History:

Received 2 October, 2018, Revised 2 August, 2019, Accepted 15 September, 2019, Published 30 September, 2019

e-ISSN: 2289-7771

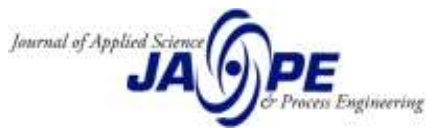


For better understanding the physics of the dielectric breakdown mechanism in a triggered discharge gap and how the dielectric breakdown is influenced by the discharge process. A three electrodes air gaps with an adjustable electrode to vary the gaps distances of the local discharge and a replaceable grounded electrode was constructed and experimentally investigated.

The paper reports the results of an investigation carried out in order to determine the critical impulse dielectric breakdown voltage. The energized electrode of the system is stressed by an impulse voltage waveform of $1.2 / 50 \mu \mathrm{s}$. The dielectric breakdown voltage variations are discussed as a function of the parameters influencing the discharge mechanism such as the gap geometry, the amplitude and polarity of the applied impulse voltage at the energized rod.

\section{Experimental setup}

The configuration of the electrical system with multiples electrodes consists of three electrodes, where the triggered electrode is a conical rod placed on the horizontal axis with the high voltage electrode an rod electrode (energized rod) facing each other together parallel with earth electrode (plane, and rods with different angles). The length of the local electric discharge can be varied by changing the position of the triggered electrode. Positive or negative electric discharges mean that the high-voltage electrode is positively or negatively energized.

The dielectric breakdown characteristics were investigated experimentally. We have accomplished several experiments by varying the main gap spacing formed between the high-voltage electrode (local electric discharge between the two horizontal conical rods) and the grounded electrode. We have studied the effect of a local electric discharge (between the energized rod and the trigger electrode) on the dielectric breakdown voltage of the main gap.

It is important to understand how the multiples gaps with local electric discharge can affect the dielectric breakdown voltage probability of the main gap with an impulse high voltage. The impulse high voltage with a waveform of $1.2 / 50 \mu \mathrm{s}$ is applied directly to the energized rod, which produced a local electric discharge between the two horizontal conical rods. All measurements and observations were carried out under standard lightning impulse voltage $(1.2 / 50 \mu \mathrm{s})$ in air at atmospheric pressure.

\section{Results and discussion}

The electrical discharge mechanism and the dielectric breakdown voltage characteristics of multiples gaps in air with the presence of local electric discharge are investigated. A high voltage impulse is applied to the energized rod electrode, which initiates the dielectric breakdown process. Local electric discharge occurring in the initial gap between the horizontal electrodes rod, results in the formation of plasma which contributes largely on the development of the others electrical discharges.

The dielectric breakdown initiation is principally due to the locally high electric fields, the field distortion and the emission mechanisms in the gap, because of the presence of local electrical discharge. The electric field is low for the dielectric breakdown of the main gap, but sufficiently for an electron to move toward the grounded electrode. Electrical discharge occurs between the energized electrode and trigger electrode and both are at the same potential. This (local electric discharge) enhances the electron emission mechanisms necessary for a development of electron avalanches. Another electric discharge forms between the local electric discharge and the grounded electrode, which breakdown the main gap.

The electric field distribution and UV radiation from the local electric discharge are the cause of the dielectric breakdown of the main gap. This is the reasons of the ionization process when the electrons are produced by the electron emission mechanisms from the local electric discharge, and the

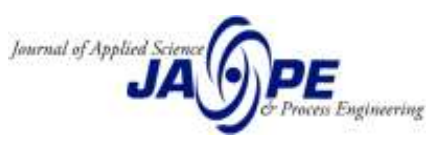


photoelectrons produced by the local electric discharge across the main gap, and after the charged particles avalanches triggered the main gap (Fig. 1). In Fig. 1 and 2 the dielectric breakdown voltage characteristics for both polarities of the local electric discharge - plane gap and the local electric discharge - rod gap (grounded conical $\operatorname{rod} \theta=15^{\circ}$ ) respectively is plotted for the trigger gap (between the energized rod and the trigger electrode) of $0.3 \mathrm{~cm}$.

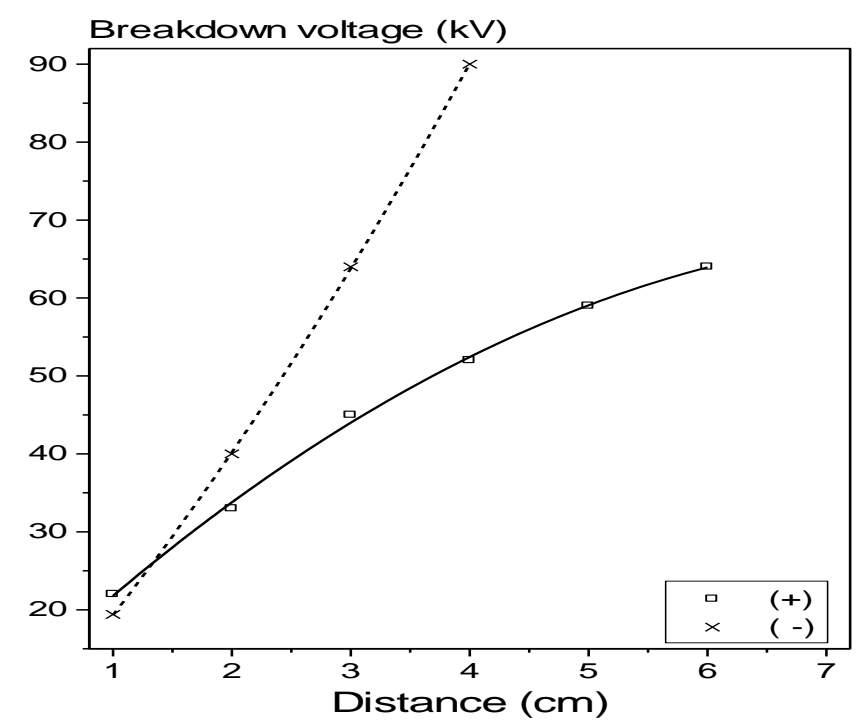

Figure 1. Dielectric breakdown voltage characteristics for both polarities of the local electric discharge - plane gap $(\mathrm{d}=0.3 \mathrm{~cm})$.

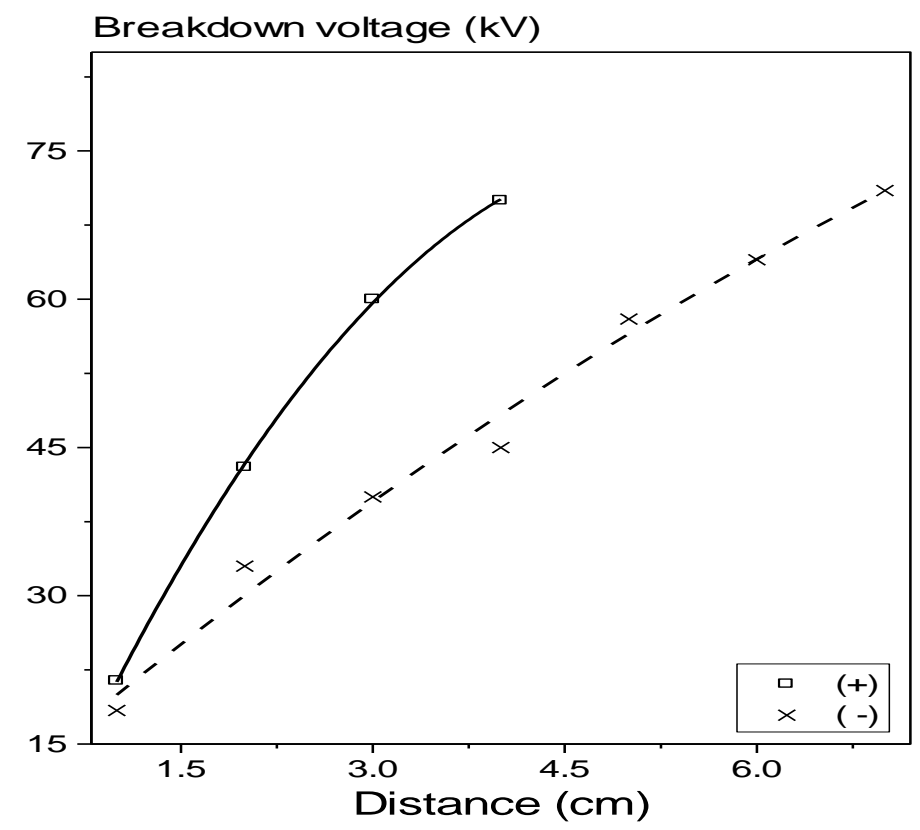

Figure 2. Dielectric breakdown voltage characteristics for both polarities of the local electric discharge - rod gap (grounded conical $\operatorname{rod} \theta=15^{\circ}$ and $\mathrm{d}=0.3 \mathrm{~cm}$ ). 
The experimental results show that the dielectric breakdown voltages for the negative polarity are significantly lower than those for the positive polarity (Fig. 2), in the local electric discharge - rod gap configuration. For the confection of the high voltage systems most of the studies are made under positive polarity which is crucial. The negative polarity is usually considered less severe; in the domain using the electrical discharges, what corresponds to it a high dielectric breakdown voltage.

We studied in a non-uniform electric field the influence of the local electric discharge on the dielectric breakdown voltage in a multiple air gaps as a function of dimensions and geometry of the gap. To study the effect of the high voltage polarities on the dielectric breakdown of the main gap in non uniform electric field, we varied the length of the local electric discharge by varying the trigger gap. In the local electric discharge - rod configuration, the negative polarity resulted in a lower dielectric breakdown voltage than those of the local electric discharge - plane configuration for all the trigger gap lengths as indicated in figures 1 through 4 . The experimental results show that the plasma formed by the local electric discharge can lower the dielectric breakdown voltage for the main gap in the local electric discharge - rod configuration for the negative polarity.

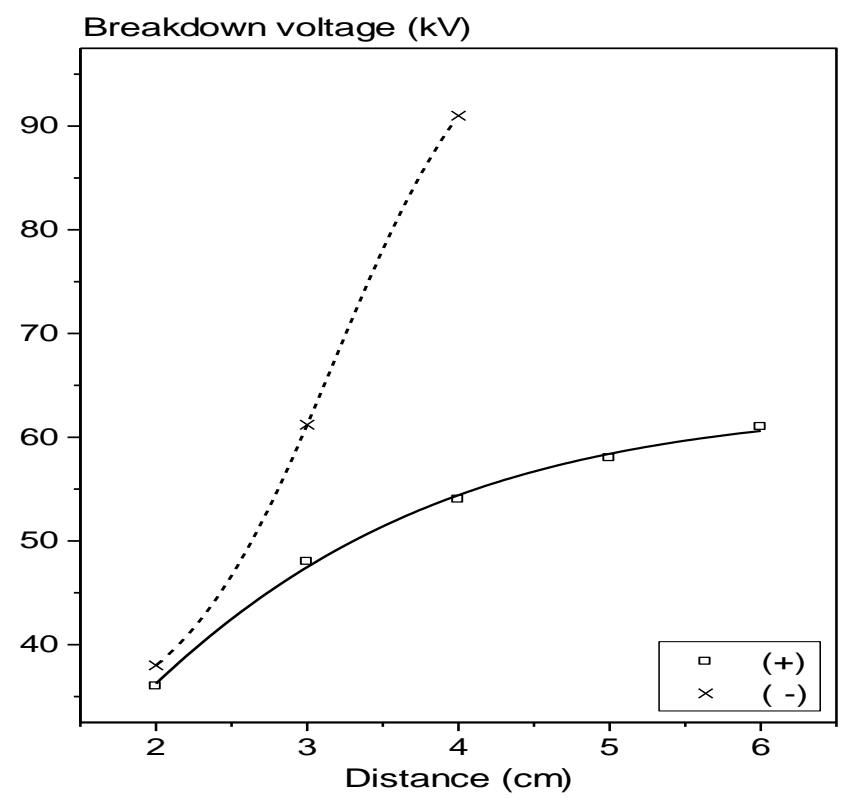

Figure 3. Dielectric breakdown voltage characteristics for both polarities of the local electric discharge - plane gap $(\mathrm{d}=2 \mathrm{~cm})$.

The high voltage insulator is an important element in the high voltage electric power transmission systems. Every imperfection of the high voltage insulators results of the rupture in the systems of the electric power transmission. The industries depend on the continuity of the power supply, and the electrical discharge is a substantial problem among the others in the electric transmission of high voltage. The flashover on the high voltage insulators is one of these problems.

The presence of the electric discharges on the high voltage insulators and their propagations on the surface of the high voltage insulator cause the flashover and the rupture of the systems. The electric discharges on the high voltage insulator trigger the dielectric breakdown of the gap between the electric high voltage line and the grounded pylon, and cause the interruption in the power supply. The polarity effect in the flashover on the high voltage insulators used in the high voltage power transmission is in the same relationship with the dielectric breakdown voltage in the presence of a local electrical discharge. 


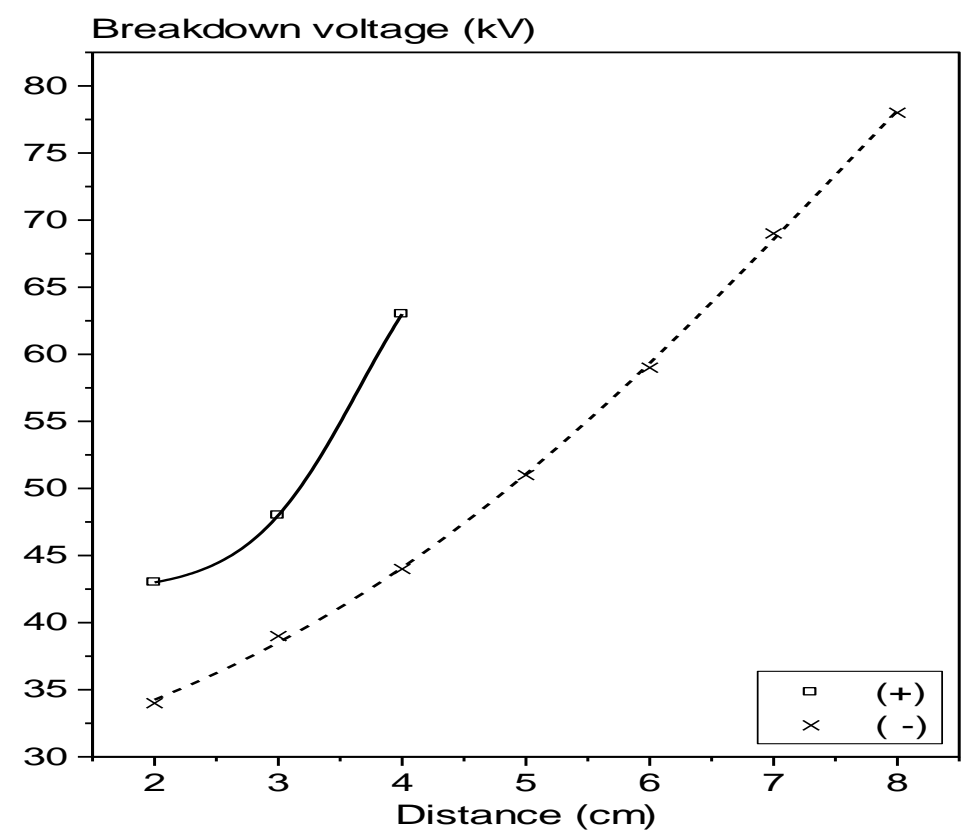

Figure 4. Dielectric breakdown characteristics for both polarities of the local electric discharge - rod gap (grounded conical $\operatorname{rod} \theta=15^{\circ}$ and $\mathrm{d}=2 \mathrm{~cm}$ ).

The cause of the voltage polarity effect in the flashover voltage of the high voltage insulators is still unclear, that the negative flashover voltage is lower than the positive flashover voltage (Fig. 5).

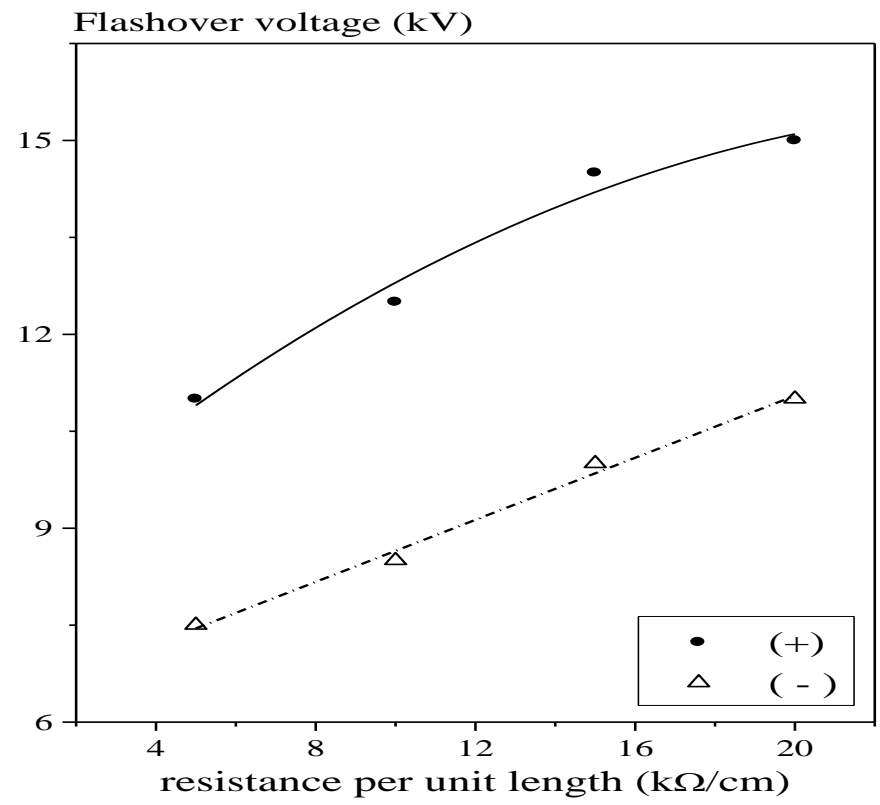

Figure 5 Characteristics of the flashover voltage for both polarities of the applied voltage. 
Several electric discharges may appear on the high voltage insulator surface after the high voltage is applied. The mechanism of the propagation of the electric discharges in the flashover of the high voltage insulators is the same of the electric discharge in a non uniform electric field of the multiples electrodes in the air gap.

The local electric discharge facilitates the development of the electrical discharges in the main gap by decreasing the breakdown voltage (Figs. 6-11). In the configuration of negative polarity of the energized electrode, the dielectric breakdown voltage of the local electric discharge - rod gap was much lower than that for air alone in the rod-rod gap (Fig. 7, Fig. 9, and Fig. 11).

The discharge process is more complex with negative polarity of the energized electrode than that with positive polarity. There are differences in the dielectric breakdown voltage of the main gap for the negative and positive electrical discharge. The difference in the dielectric breakdown process between positive and negative impulse voltage can not be described only by using the applied electric field distribution in the gap. The effects of the space charge generated at the local electrical discharge must be included in the analysis [11]. Space charge will be generated around the local electric discharge and adjacent to the rod electrode, for the local electrode discharge - rod geometry, because the highest electric field is located both adjacent to the local electric discharge electrode and the grounded rod.

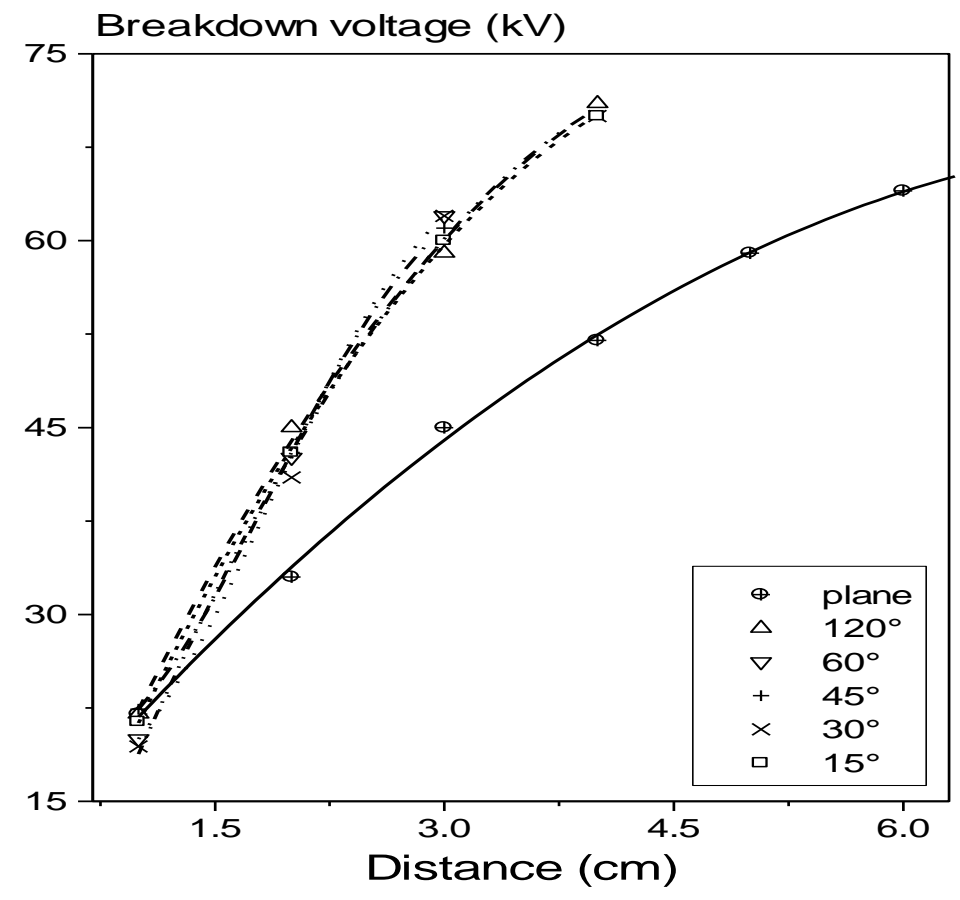

Figure 6. Dielectric breakdown voltage characteristics for positive polarity of the local electric discharge (different geometries and $\mathrm{d}=0.3 \mathrm{~cm}$ ).

The electric field distribution between the positive space charge and the negative space charge reduces the mean electric field strength in the main gap [12]. This configuration is similar to the rod rod gap configuration, which has a higher dielectric breakdown voltage than a rod - plate electrode configuration. Under dynamic conditions for the local electric discharge - plane geometry, the highest electric field strength is located at the local electric discharge. It is therefore reasonable to assume that the dielectric breakdown process starts at the local electric discharge with positive polarity and propagates towards the ground plane electrode. 


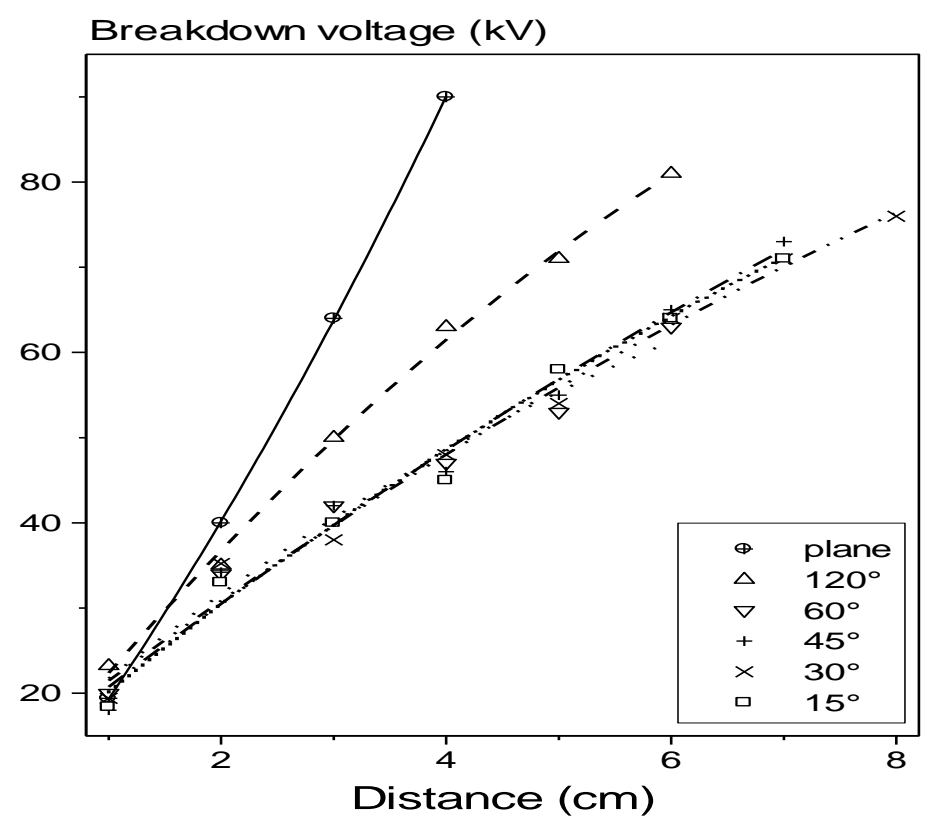

Figure 7. Dielectric breakdown characteristics for negative polarity of the local electric discharge (different geometries and $\mathrm{d}=0.3 \mathrm{~cm}$ ).

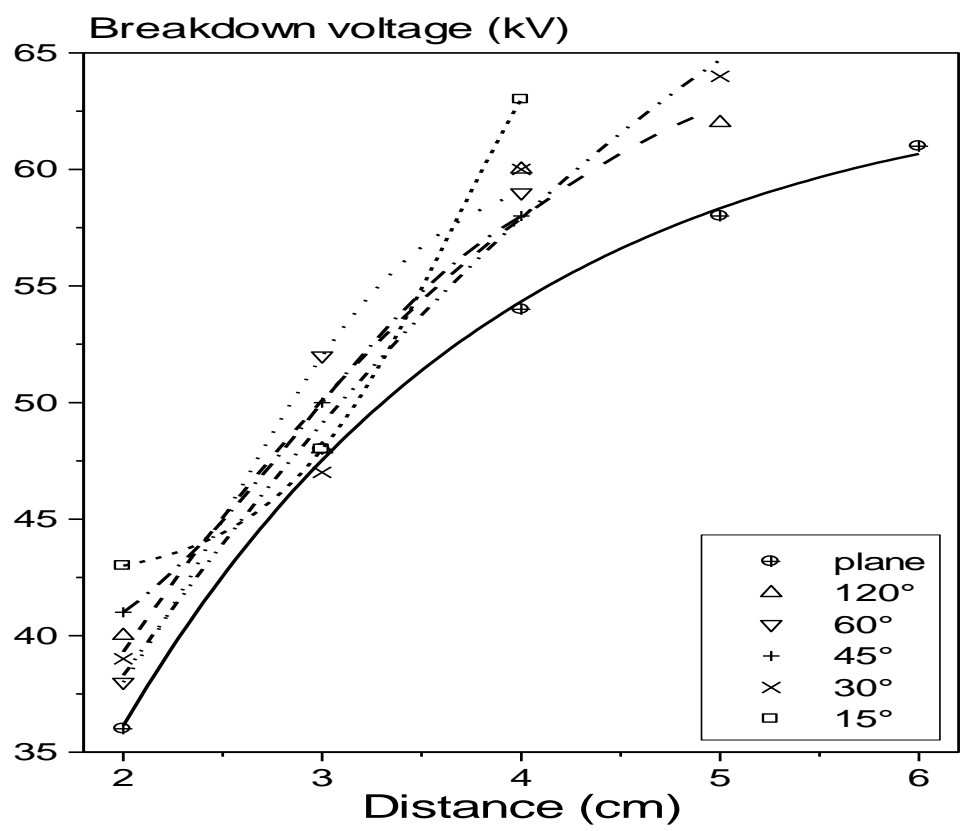

Figure 8. Dielectric breakdown characteristics for positive polarity of the local electric discharge (different geometries and $\mathrm{d}=2 \mathrm{~cm}$ ).

The trigger electrode stipulating a local electric discharge which serves as source for the development of streamers when high voltages are applied. From the local plasma, streamers initiate electron avalanche that bridge the gaps to the high voltage electrode and ground electrode, providing a 
closing path for the dielectric breakdown of the main gap. The dielectric breakdown of the main gap is caused partly with positive and negative streamers. The breakdown voltage decreases when we increase the gap of the local electric discharge (Figs. 6-11). With a local electric discharge the dielectric breakdown mechanism starts at the electric discharge electrode that influences the emergence and development of electrical discharge from the local electric discharge.

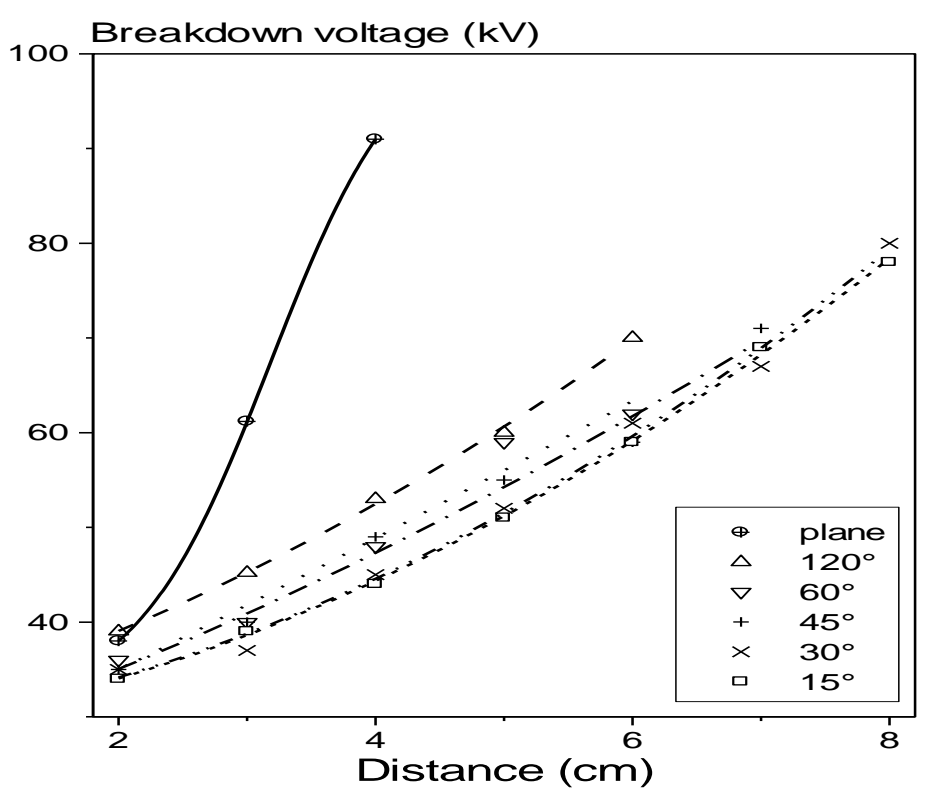

Figure 9. Dielectric breakdown characteristics for negative polarity of the local electric discharge (different geometries and $\mathrm{d}=2 \mathrm{~cm}$ ).

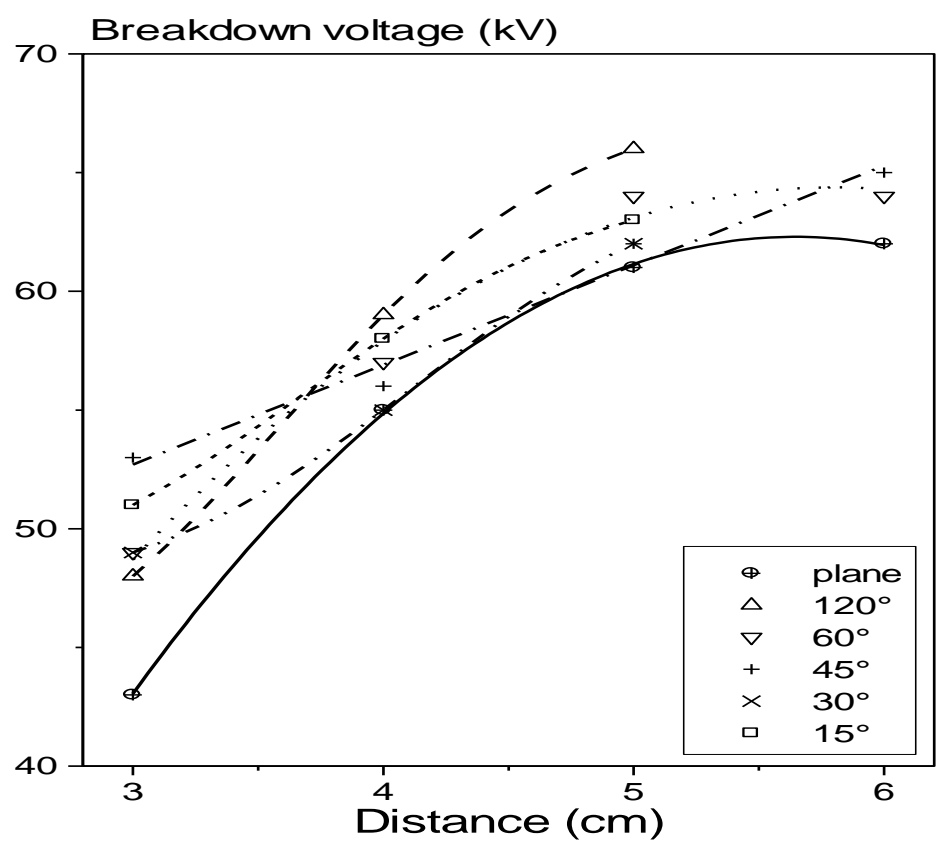

Figure 10. Dielectric breakdown characteristics for positive polarity of the local electric discharge (different geometries and $\mathrm{d}=3 \mathrm{~cm}$ ). 
The trigger electrode providing a local electric discharge which serves as source for the development of streamers when high voltages are applied. From the local plasma, streamers initiate electron avalanche that bridge the gaps to the high voltage electrode and ground electrode, providing a closing path for the dielectric breakdown of the main gap. The dielectric breakdown of the main gap is caused partly with positive and negative streamers. The breakdown voltage decreases when we increase the gap of the local discharge (Figs. 6-11). With a local discharge the dielectric breakdown mechanism starts at the discharge electrode that influences the emergence and development of discharge from the local discharge.

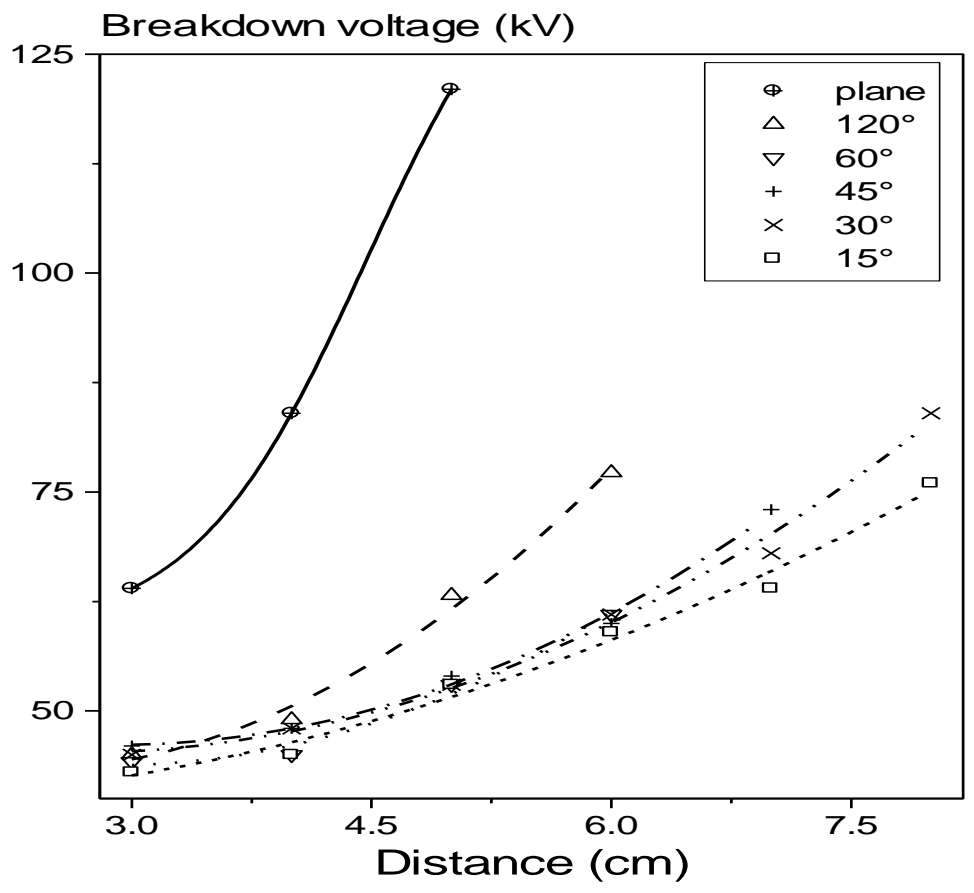

Figure 11. Dielectric breakdown characteristics for negative polarity of the local discharge (different geometries and $\mathrm{d}=3 \mathrm{~cm}$ ).

In the presence of a local electric discharge, the dielectric breakdown initiation in the main gap is principally due to the following mechanisms: the electric field distortion with the space charged particles and the photon emission due to the existing plasma channel of the local electric discharge. The polarity effect on the dielectric breakdown of the main gap can be related to the electric field distortion.

Triggered dielectric breakdown with local electric discharge can develop in an electric field lower than the required for dielectric breakdown without local electric discharge. The local electric discharge creates plasma which can be propagated and ionized the air in electric fields lower than those required for dielectric breakdown without initial local electric discharge. The local electric discharge creates plasma which distorts the electric field due to the high density of the charged particles in the volume.

The reduction of the dielectric breakdown voltage with local electric discharge makes this process so attractive. As a local electric discharge is triggered ionizing a volume of gas, the streamer can develop with electric fields lower than the dielectric breakdown voltage without a local electric discharge. The physical mechanisms depend strongly on polarity, electrode shape, distance and properties of the applied voltage, which shows that the propagation of discharges in air is a highly 
complex process, which makes the scientific progress so difficult in this domain. To understand the mechanisms of the triggered a main gap with a local electrical discharge, we must know the electric field distribution under the static condition and the electric field distribution under the dynamic condition, taking into account the voltage polarity. It is significant to note that the geometry configuration of the gap plays an important role in the dynamic process of the development of the electric discharge in the multiples electrodes.

\section{Conclusion}

Electrical discharges in gases present a considerable interest for a large range of applications. In a multiple gaps system with a high voltage electrode surrounded by a several grounded electrodes, the dielectric breakdown can occur in longer gap spacing. In order to study the electric discharge mechanism, the characteristics of dielectric breakdown voltage for the air gas in the presence of a local electric discharge with miltiple electrodes were investigated.

The performance of various geometries of the electrodes configurations in air gaps has been studied experimentally. These electrode configurations are important in electric power distribution systems; the shape of electrodes has a significant impact on the dielectric breakdown voltages of air gaps. Experimental researches were undertaken with different geometries at atmospheric pressure under impulse voltage.

The dielectric breakdown voltage characteristic of air gap is affected by the surrounding conditions. The emission and photoemission intensity of the local electric discharge have an important role in the propagation of the local electric discharge to dielectric breakdown the main gap. The dielectric breakdown voltage of the main gap depends on the electrical field distribution near the local discharge.

\section{References}

[1] Xiong, Z., Kushner, M.J. (2011). Photo-triggering and Secondary Electron Produced Ionization in Electric Discharge ArF* Excimer Lasers. J. Appl. Phys., Vol. 110, 083304.

[2] Cheng, X.B., Liu, J.L., Qian, B.L., Chen, Z., Feng, J.H. (2010). Research of a High-Current Repetitive Triggered Spark-Gap Switch and its Application. IEEE Trans. Plasma Sci., Vol. 38(3), 516-522.

[3] Li, L., Li, C., Xiangdong, Q., Fuchang, L., Yuan, P. (2012). Modeling of Switching Delay in Gas-Insulated Trigatron Spark Gaps. J. Appl. Phys., Vol. 111, 053306.

[4] Negara, Y., Yaji, K., Imasaka, K., Hayashi, N., Suehiro, J., Hara, M. (2007). AC Particle-Triggered Corona Discharge in Low Pressure SF6 Gas. IEEE Trans. Diel. Elect. Ins., Vol. 14(1), 91-100.

[5] Sharma, R.K., Chavan, S.G., Sadhu, R.K., Bhattacharya, S., Srivastava, G.P. (2013). Experimental Study of Flat Format Multichannel Triggered Rail Spark Gap. IEEE trans. on Plasma Sci., Vol. 41(10), 2666-2670.

[6] Forestier, B., Houard, A., Revel, I., Durand, M., André, Y.B., Prade, B., Jarnac, A., Carbonnel, J., Le Nevé, M., de Miscault, J.C., Esmiller, B., Chapuis, D., Mysyrowicz, A. (2012). Triggering, Guiding and Deviation of Long Air Spark Discharges with Femtosecond Laser Filament. AIP Advances, Vol. 2, 012151.

[7] Kikuchi, J., Suzuki, Y., Muto, T., Ibuka, S., Ishii, S. (2012). Effect of DC Pre-Discharge on the Generation of Atmospheric Pulsed Microdischarges. Japanese J. Appl. Phys., Vol. 51, 046001.

[8] Niermann, B., Budunoglu, I.L., Gurel, K., Boke, M., Ilday, F.O., Winter, J. (2012). Application of a Modelocked Fiber Laser for Highly Time Resolved Broadband Absorption Spectroscopy and Laser-Assisted Breakdown on Micro-plasmas. J. Phys. D: Appl. Phys., Vol. 45, 245202.

[9] Hara, M., Negara, Y., Setoguchi, M., Kurihara, T., Suehiro, J., Hayashi, N. (2005). Particle-triggered Prebreakdown Phenomena in Atmospheric Air Gap under ac Voltage. IEEE Trans. Diel. Elect. Ins., Vol. 12(5), 1071-1081.

[10] Schoenbach, K., Kolb, J., Xiao, S., Katsuki, S., Minamitani, Y., Joshi, R. (2008). Electrical Breakdown of Water in Microgaps. Plasma Sources Sci. Technol., Vol. 17, 024010. 
[11] Settaouti, A. (2010). Monte Carlo Simulation of Avalanche Formation and Streamer Discharge., Electr. Eng., Vol. 92, 35-42.

[12] Settaouti, A., Settaouti, L. (2011). Monte Carlo Simulation of Electrical Corona Discharge in Air. Elect. Pow. Syst. Res., Vol. 81, 84-89. 